\title{
GENERALIZED DERIVATIONS ACTING AS HOMOMORPHISM OR ANTI-HOMOMORPHISM WITH CENTRAL VALUES IN SEMIPRIME RINGS
}

\author{
B. DHARA, S. KAR, AND K. G. PRADHAN
}

Received 21 January, 2015

\begin{abstract}
Let $R$ be a semiprime ring with center $Z(R)$. A mapping $F: R \rightarrow R$ is called a generalized derivation if there exists a derivation $d: R \rightarrow R$ such that $F(x y)=F(x) y+x d(y)$ holds for all $x, y \in R$. In the present paper, our main object is to study the situations: (1) $F(x y)-F(x) F(y) \in Z(R)$, (2) $F(x y)+F(x) F(y) \in Z(R)$, (3) $F(x y)-F(y) F(x) \in Z(R)$, (4) $F(x y)+F(y) F(x) \in Z(R)$; for all $x, y$ in some suitable subset of $R$.
\end{abstract}

2010 Mathematics Subject Classification: 16W25;; 16R50; 16N60

Keywords: semiprime ring, derivation, generalized derivation

\section{INTRODUCTION}

Let $R$ be an associative ring with center $Z(R)$. For $x, y \in R,[x, y]$ denotes the commutator element $x y-y x$. We use the notation to define the Engel type polynomial $[x, y]_{n+1}=\left[[x, y]_{n}, y\right]$ instead of $[x, y, y, \ldots, y]$ for $n \geq 1$ and $[x, y]_{1}=[x, y]$. Recall that a ring $R$ is called prime if for any $a, b \in R, a R b=(0)$ implies that either $a=0$ or $b=0$ and is called semiprime if for any $a \in R, a R a=(0)$ implies $a=0$. An additive mapping $F: R \rightarrow R$ is called a generalized derivation of $R$ if there exists a derivation $d: R \rightarrow R$ such that $F(x y)=F(x) y+x d(y)$ holds for any $x, y \in R$. If $d=0$, then $F$ is said to be a left centralizer map of $R$. For any subset $S$ of $R$, $r_{R}(S)$ denotes the right annihilator of $S$ in $R$, that is, $r_{R}(S)=\{x \in R \mid S x=0\}$ and $l_{R}(S)$ denotes the left annihilator of $S$ in $R$ that is, $l_{R}(S)=\{x \in R \mid x S=0\}$. If $r_{R}(S)=l_{R}(S)$, then $r_{R}(S)$ is called an annihilator ideal of $R$ and is written as $a n n_{R}(S)$.

Let $S$ be a nonempty subset of a ring $R$. The mapping $F: R \rightarrow R$ is said to be a homomorphism ( anti-homomorphism) acting on $S$ if $F(x y)=F(x) F(y)$ holds for all $x, y \in S$ (respectively $F(x y)=F(y) F(x)$ holds for all $x, y \in S$ ).

This research is supported by a grant from National Board for Higher Mathematics (NBHM), India. Grant No. is NBHM/R.P. 26/ 2012/Fresh/1745 dated 15.11.12. 
A series of papers in literature studied the homomorphism or anti-homomorphism of some specific type of additive mappings in prime and semiprime rings under certain conditions (see [1-4, 7, 10-12,14,15]).

In [4], Bell and Kappe showed that if a derivation $d$ of a prime ring $R$ can act as homomorphism or anti-homomorphism on a nonzero right ideal of $R$, then $d=0$ on $R$. Then Ali, Rehman and Ali in [2] proved a similar result to Lie ideal case. They proved that if $R$ is a 2-torsion free prime ring, $L$ a nonzero Lie ideal of $R$ such that $u^{2} \in L$ for all $u \in L$ and $d$ acts as a homomorphism or anti-homomorphism on $L$, then either $d=0$ or $L \subseteq Z(R)$.

On the other hand, the authors developed above results, replacing the derivation $d$ with a generalized derivation $F$ of $R$. In this view, Rehman [14] proved the following:

Let $R$ be a 2-torsion free prime ring and $I$ be a nonzero ideal of $R$. Suppose $F: R \rightarrow R$ is a nonzero generalized derivation with $d$.

(i) If $F$ acts as a homomorphism on $I$ and if $d \neq 0$, then $R$ is commutative.

(ii) If $F$ acts as an anti-homomorphism on $I$ and if $d \neq 0$, then $R$ is commutative.

Recently, in [3] Ali and Huang studied the case when a generalized Jordan $(\alpha, \beta)$ derivation $F$ acts as homomorphism or anti-homomorphism on a square closed Lie ideal $U$ in prime ring $R$.

It is natural to investigate the above situations in semiprime rings. Recently, in [7] the first author of this article has studied the situations, when a generalized derivation $F$ of a semiprime ring $R$ acts as homomorphism or anti-homomorphism in a nonzero left ideal of $R$.

From above results, it is natural to consider the situations, when the generalized derivations $F$ satisfies the identities: (1) $F(x y)-F(x) F(y) \in Z(R),(2) F(x y)+$ $F(x) F(y) \in Z(R)$, (3) $F(x y)-F(y) F(x) \in Z(R)$, (4) $F(x y)+F(y) F(x) \in Z(R)$; for all $x, y$ in some suitable subset of $R$.

Recently, Albas [1] studied the above mentioned identities in prime rings. Albas proved the following theorems:

Theorem A. Let $R$ be a prime ring with center $Z(R)$ and $I$ be a nonzero ideal of $R$. If $R$ admits a nonzero generalized derivation $F$ of $R$, with associated derivation d such that $F(x y)-F(x) F(y) \in Z(R)$ or $F(x y)+F(x) F(y) \in Z(R)$ for all $x, y \in I$, then either $R$ is commutative or $F=I_{i d}$ or $F=-I_{i d}$, where $I_{i d}$ denotes the identity map of the ring $R$.

Theorem B. Let $R$ be a prime ring with center $Z(R)$ and $I$ be a nonzero ideal of $R$. If $R$ admits a nonzero generalized derivation $F$ of $R$, with associated derivation $d$ such that $F(x y)-F(y) F(x) \in Z(R)$ or $F(x y)+F(y) F(x) \in Z(R)$ for all $x, y \in I$, then $R$ is commutative. 
In the present paper our main object is to investigate the situations in semiprime rings.

\section{PRELIMINARIES}

We shall use following basic identities which will be used frequently: for $x, y, z \in$ $R$,

$$
[x y, z]=x[y, z]+[x, z] y \quad \text { and } \quad[x, y z]=y[x, z]+[x, y] z .
$$

We need the following facts which will be used to prove our theorems:

Fact-1. [5, Theorem 3] Let $R$ be a semiprime ring and $U$ a nonzero left ideal of $R$. If $R$ admits a derivation $d$ which is nonzero on $U$ and $[d(x), x] \in Z(R)$ for all $x \in U$, then $R$ contains a nonzero central ideal.

Fact-2. [8, Fact-4]Let $R$ be a semiprime ring, $d$ a nonzero derivation of $R$ such that $x[[d(x), x], x]=0$ for all $x \in R$. Then $d$ maps $R$ into its center.

Fact-3. [13, Corollary 2] If $R$ is a semiprime ring and $I$ is an ideal of $R$, then $I \cap \operatorname{ann}_{R}(I)=0$.

Fact-4. [6, Lemma 2] (a) If $R$ is a semiprime ring, the center of a nonzero onesided ideal is contained in the center of $R$; in particular, any commutative one-sided ideal is contained in the center of $R$.

(b) If $R$ is a prime ring with a nonzero central ideal, then $R$ must be commutative.

Fact-5. [9, Corollary 2.6] Let $R$ be a prime ring, $I$ a nonzero ideal of $R$ and $F: R \rightarrow R$ a nonzero left centralizer map. (1) If $F(x) F(y)-F(x y) \in Z(R)$ for all $x, y \in I$, then either $R$ is commutative or $F(r)=r$ for all $r \in R$. (2) If $F(x) F(y)+$ $F(x y) \in Z(R)$ for all $x, y \in I$, then either $R$ is commutative or $F(r)=-r$ for all $r \in R$.

\section{MAin RESUlts}

Theorem 1. Let $R$ be a semiprime ring with center $Z(R)$ and $I$ a nonzero ideal of $R$. Let $F: R \rightarrow R$ be a generalized derivation associated with the derivation $d: R \rightarrow R$. If $F(x y)-F(x) F(y) \in Z(R)$ for all $x, y \in I$, then one of the following holds:

(1) $R$ contains a nonzero central ideal;

(2) $d(I)=(0)$ and $F$ is a left centralizer map on $I$ such that $[F(x), x]=0$ for all $x \in I$.

Proof. By our assumption, we have

$$
F(x y)-F(x) F(y) \in Z(R)
$$


for all $x, y \in I$. Replacing $y$ with $y z$, where $z \in I$, we get

$$
F(x y z)-F(x) F(y z) \in Z(R)
$$

which implies

$$
F(x y) z+x y d(z)-F(x)\{F(y) z+y d(z)\} \in Z(R)
$$

that is

$$
(F(x y)-F(x) F(y)) z+(x-F(x)) y d(z) \in Z(R) .
$$

Commuting both sides with $z$, we get

$$
[(F(x y)-F(x) F(y)) z+(x-F(x)) y d(z), z]=0
$$

for all $x, y, z \in I$. By using (3.1), above relation yields

$$
[(x-F(x)) y d(z), z]=0
$$

for all $x, y, z \in I$. Now we put $x=x z$, and then obtain that

$$
[(x z-F(x) z-x d(z)) y d(z), z]=0
$$

which is

$$
[(x-F(x)) z y d(z), z]-[x d(z) y d(z), z]=0
$$

for all $x, y, z \in I$. In (3.6), replacing $y$ with $z y$, we get

$$
[(x-F(x)) z y d(z), z]=0
$$

for all $x, y, z \in I$. Using (3.9), (3.8) implies

$$
[x d(z) y d(z), z]=0
$$

for all $x, y, z \in I$. Now we put $x=d(z) x$ in (3.10), and then we see that

$$
0=[d(z) x d(z) y d(z), z]=d(z)[x d(z) y d(z), z]+[d(z), z] x d(z) y d(z)
$$

for all $x, y, z \in I$. As an application of (3.10), (3.11) reduces to

$$
[d(z), z] x d(z) y d(z)=0
$$

for all $x, y, z \in I$. Replacing $x$ with $x z$ and $y$ with $z y$ respectively in (3.12), we get

$$
[d(z), z] x z d(z) y d(z)=0
$$

and

$$
[d(z), z] x d(z) z y d(z)=0
$$

for all $x, y, z \in I$. Subtracting one from another yields

$$
[d(z), z] x[d(z), z] y d(z)=0
$$

for all $x, y, z \in I$. Replacing $y$ with $y z$ in (3.15) and right multiplying (3.15) by $z$ respectively and then subtracting one from another yields

$$
[d(z), z] x[d(z), z] y[d(z), z]=0
$$


for all $x, y, z \in I$, which implies $(I[d(z), z])^{3}=(0)$ for all $z \in I$. Since $R$ is semiprime, it contains no nonzero nilpotent left ideal, implying $I[d(z), z]=(0)$ for all $z \in I$. Thus, $[d(z), z] \in A n n_{R}(I)$ for all $z \in I$. Since $I$ is an ideal, we conclude that $[d(z), z] \in I$ for all $z \in I$. This implies that $[d(z), z] \in I \cap A n n_{R}(I)$ for all $z \in I$. In view of Fact-3, $[d(z), z]=0$ for all $z \in I$. Further, if $d$ is derivation such that $d(I) \neq(0)$, then by Fact- $1, R$ contains a nonzero central ideal.

Let $d(I)=(0)$. Then $F(x y)=F(x) y+x d(y)=F(x) y$ for all $x, y \in I$, i.e., $F$ is a left centralizer map on $I$. Then by our hypothesis, we have

$$
F(x)(y-F(y)) \in Z(R)
$$

for all $x, y \in I$. Replacing $y$ with $y u$, where $u \in I$, we get

$$
F(x)(y-F(y)) u \in Z(R)
$$

for all $x, y, u \in I$. Commuting both sides with $v$, where $v \in I$, we get

$$
F(x)(y-F(y)) u v-v F(x)(y-F(y)) u=0 .
$$

By using (3.18), it reduces to

$$
v F(x)(y-F(y)) u \in Z(R)
$$

for all $u, v, x, y \in I$. We choose $x, y \in I$ such that $a=F(x)(y-F(y)) \neq 0$. Then from above, we have $I a I \subseteq Z(R)$, that is, $R$ contains a central ideal. If this ideal is zero ideal, then

$$
I(F(x)(y-F(y)))=(0)
$$

for all $x, y \in I$. Replacing $x$ with $x z, z \in I$, this gives

$$
I(F(x) z(y-F(y)))=(0)
$$

for all $x, y, z \in I$. Thus $F(x) z(y-F(y)) \in I \cap a n n_{R}(I)=(0)$ for all $x, y, z \in I$. This gives

$$
[F(x), x] z(y-F(y))=0
$$

for all $x, y, z \in I$. Putting $y=y^{2}$ and $z=z y$ respectively and then subtracting one from another, we get

$$
[F(x), x] z[F(y), y]=0
$$

for all $x, y, z \in I$. Since $I$ is an ideal of $R$, it follows that $([F(x), x] I)^{2}=(0)$ for all $x \in I$. Since semiprime ring contains no nonzero nilpotent ideal, we have $[F(x), x] I=(0)$ for all $x \in I$. Thus by Fact-3, $[F(x), x] \in I \cap a n n_{R}(I)=(0)$ for all $x \in I$. Thereby, the proof is completed.

Theorem 2. Let $R$ be a semiprime ring with center $Z(R)$ and $I$ a nonzero ideal of $R$. Let $F: R \rightarrow R$ be a generalized derivation associated with the derivation $d: R \rightarrow R$. If $F(x y)+F(x) F(y) \in Z(R)$ for all $x, y \in I$, then one of the following holds:

(1) $R$ contains a nonzero central ideal; 

$x \in I$.

$(2) d(I)=(0)$ and $F$ is a left centralizer map on $I$ such that $[F(x), x]=0$ for all

Proof. If we replace $F$ with $-F$ and $d$ with $-d$ in Theorem 1, we conclude that $(-F)(x y)-(-F)(x)(-F)(y) \in Z(R)$ for all $x, y \in I$, implies $[(-d)(x), x]=0$ for all $x \in I$, that is, $F(x y)+F(x) F(y) \in Z(R)$ for all $x, y \in I$, implies $[d(x), x]=0$ for all $x \in I$. Hence conclusion follows by Theorem 1 .

The following corollary is immediate consequences of the Theorem 1 and Theorem 2 by using Fact- 4 and Fact- 5 .

Corollary 1. Let $R$ be a prime ring with center $Z(R)$ and $F: R \rightarrow R$ be a generalized derivation associated with the derivation $d: R \rightarrow R$.

(1) If $R$ satisfies $F(x y)+F(x) F(y) \in Z(R)$, then either $R$ is commutative or $F(x)=-x$ for all $x \in R$.

(2) If $R$ satisfies $F(x y)-F(x) F(y) \in Z(R)$, then either $R$ is commutative or $F(x)=x$ for all $x \in R$.

Theorem 3. Let $R$ be a semiprime ring with center $Z(R)$. Let $F: R \rightarrow R$ be a generalized derivation associated with the derivation $d: R \rightarrow R$. If $F(x y)-$ $F(y) F(x) \in Z(R)$ for all $x, y \in R$, then one of the following holds: (1) $R$ contains a nonzero central ideal; (2) $F$ is a left centralizer map of $R$ such that $F: R \rightarrow Z(R)$.

Proof. By hypothesis, we have

$$
F(x y)-F(y) F(x) \in Z(R)
$$

for all $x, y \in R$. Putting $x=x z$, we have

$$
F(x z y)-F(y)(F(x) z+x d(z)) \in Z(R)
$$

which gives

$$
F(x) z y+x d(z y)-F(y) F(x) z-F(y) x d(z) \in Z(R) .
$$

Commuting both sides with $z$, we have

$$
[F(x) z y-F(y) F(x) z-F(y) x d(z)+x d(z y), z]=0
$$

that is

$$
[F(x) z y, z]-[F(y) F(x), z] z-[F(y) x d(z)-x d(z y), z]=0
$$

for all $x, y, z \in R$. From (3.21), we can write that $[F(x y)-F(y) F(x), z]=0$ for all $x, y, z \in R$, that is, $[F(x y), z]=[F(y) F(x), z]$ for all $x, y, z \in R$. Thus (3.25) reduces to

$$
[F(x) z y, z]-[F(x y), z] z-[F(y) x d(z)-x d(z y), z]=0
$$

for all $x, y, z \in R$. Putting $y=z^{2}$ in (3.26), we have

$$
\left[F(x) z^{3}, z\right]-\left[F(x) z^{2}+x d\left(z^{2}\right), z\right] z-\left[(F(z) z+z d(z)) x d(z)-x d\left(z^{3}\right), z\right]=0
$$


that is,

$$
\left[F(z) z x d(z)+z d(z) x d(z)-x d\left(z^{3}\right)+x d\left(z^{2}\right) z, z\right]=0
$$

for all $x, z \in R$. Putting $x=z x$ in (3.26), we have

$[(F(z) x+z d(x)) z y, z]-[F(z) x y+z d(x y), z] z-[F(y) z x d(z)-z x d(z y), z]=0$

that is,

$$
\begin{aligned}
{[F(z) x z y, z]-[} & {[F(z) x y, z] z-} \\
& {[F(y) z x d(z)-z x d(z y)-z d(x) z y+z d(x y) z, z]=0 }
\end{aligned}
$$

for all $x, y, z \in R$. Assuming $y=z$, we have

$$
\left[F(z) z x d(z)-z x d\left(z^{2}\right)-z d(x) z^{2}+z d(x z) z, z\right]=0
$$

for all $x, z \in R$. Subtracting (3.31) from (3.28), we get

$$
\left[z d(z) x d(z)-x d\left(z^{3}\right)+x d\left(z^{2}\right) z+z x d\left(z^{2}\right)+z d(x) z^{2}-z d(x z) z, z\right]=0
$$

for all $x, z \in R$. This reduces to

$$
[z d(z) x d(z), z]+\left[-x d\left(z^{3}\right)+x d\left(z^{2}\right) z+z x d\left(z^{2}\right)-z x d(z) z, z\right]=0
$$

for all $x, z \in R$. Now putting $x=z x$ in (3.33), we get

$$
[z d(z) z x d(z), z]+z\left[-x d\left(z^{3}\right)+x d\left(z^{2}\right) z+z x d\left(z^{2}\right)-z x d(z) z, z\right]=0
$$

for all $x, z \in R$. Left multiplying (3.33) by $z$ and then subtracting from (3.34), we get

$$
[z[d(z), z] x d(z), z]=0
$$

for all $x, z \in R$. Again putting $x=x z$ in above relation, we get

$$
[z[d(z), z] x z d(z), z]=0
$$

for all $x, z \in R$. Now right multiplying (3.35) by $z$ and then subtracting from (3.36), we obtain

$$
[z[d(z), z] x[d(z), z], z]=0
$$

and hence

$$
[z[d(z), z] x z[d(z), z], z]=0
$$

for all $x, z \in R$. This implies

$$
z[d(z), z] x z[d(z), z] z-z^{2}[d(z), z] x z[d(z), z]=0
$$

for all $x, z \in R$. In (3.39), replacing $x$ with $x z[d(z), z] u$, we obtain

$$
z[d(z), z] x z[d(z), z] u z[d(z), z] z-z^{2}[d(z), z] x z[d(z), z] u z[d(z), z]=0
$$

for all $x, u, z \in R$. Using (3.39), (3.40) gives

$$
z[d(z), z] x z^{2}[d(z), z] u z[d(z), z]-z[d(z), z] x z[d(z), z] z u z[d(z), z]=0
$$


that is

$$
z[d(z), z] x[z[d(z), z], z] u z[d(z), z]=0
$$

for all $x, u, z \in R$. This implies $[z[d(z), z], z] x[z[d(z), z], z] u[z[d(z), z], z]=0$ for all $x, u, z \in R$, which is $(R[z[d(z), z], z])^{3}=(0)$ for all $z \in R$. Since $R$ is semiprime, we conclude that $R[z[d(z), z], z]=(0)$ for all $z \in R$. Hence, $z[[d(z), z], z]=0$ for all $z \in R$. Then by Fact-2, either $d(R)=(0)$ or $d(R) \subseteq Z(R)$. If $d(R) \neq(0)$, then the second case implies $[d(x), x]=0$ for all $x \in R$. Hence in view of Fact-1, $R$ contains a nonzero central ideal.

Let $d(R)=(0)$. Then $F(x y)=F(x) y+x d(y)=F(x) y$ for all $x, y \in R$, i.e., $F$ is a left centralizer map of $R$. Then by our hypothesis, we have

$$
F(x) y-F(y) F(x) \in Z(R)
$$

for all $x, y \in R$. Replacing $y$ with $y u$, where $u \in R$, we get

$$
F(x) y u-F(y) u F(x) \in Z(R)
$$

that is

$$
(F(x) y-F(y) F(x)) u+F(y)[F(x), u] \in Z(R)
$$

for all $x, y, u \in R$. Commuting both sides with $u$, we get

$$
[(F(x) y-F(y) F(x)) u, u]+[F(y)[F(x), u], u]=0
$$

for all $x, y, u \in R$. Since $F(x) y-F(y) F(x) \in Z(R)$ for all $x, y \in R$, we have from (3.46) that

$$
[F(y)[F(x), u], u]=0
$$

for all $x, y, u \in R$. We put $y=y r$ in above and get

$$
[F(y) r[F(x), u], u]=0
$$

for all $x, y, u, r \in R$. Now putting $y=y u$ in above, we have

$$
[F(y) u r[F(x), u], u]=0
$$

for all $x, y, u, r \in R$. Left multiplying (3.48) by $u$, we get

$$
[u F(y) r[F(x), u], u]=0
$$

for all $x, y, u, r \in R$. Subtracting (3.50) from (3.49), we obtain that

$$
[[F(y), u] r[F(x), u], u]=0
$$

for all $x, y, u, r \in R$. In particular, above relation yields

$$
[[F(x), u] r[F(x), u], u]=0
$$

that is

$$
[F(x), u] r[F(x), u] u-u[F(x), u] r[F(x), u]=0
$$

for all $x, u, r \in R$. In (3.53), replacing $r$ with $r[F(x), u] v$ we get

$$
[F(x), u] r[F(x), u] v[F(x), u] u-u[F(x), u] r[F(x), u] v[F(x), u]=0 .
$$


By using (3.53), (3.54) becomes

$$
[F(x), u] r u[F(x), u] v[F(x), u]-[F(x), u] r[F(x), u] u v[F(x), u]=0,
$$

which is

$$
[F(x), u] r[[F(x), u], u] v[F(x), u]=0
$$

for all $x, r, u, v \in R$. Replacing $v$ with $v u$ in (3.56) and right multiplying (3.56) by $u$ respectively and then subtracting one from another, we have

$$
[F(x), u] r[[F(x), u], u] v[[F(x), u], u]=0 .
$$

Similarly, just from above relation, we can write

$$
[[F(x), u], u] r[[F(x), u], u] v[[F(x), u], u]=0 .
$$

Thus $([[F(x), u], u] R)^{3}=(0)$ for all $x, u \in R$. Since $R$ is semiprime, $R$ contains no nonzero nilpotent ideals. Hence $[[F(x), u], u] R=(0)$ and so $[[F(x), u], u]=0$ for all $x, u \in R$. Then by Fact-1, we conclude that either $[F(x), u]=0$ for all $x, u \in R$ or $R$ contains a nonzero central ideal. If $[F(x), u]=0$ for all $x, u \in R$, then $F$ maps $R$ into its center. Thus we obtain our all conclusions.

Theorem 4. Let $R$ be a semiprime ring with center $Z(R)$. Let $F: R \rightarrow R$ be a generalized derivation associated with the derivation $d: R \rightarrow R$. If $F(x y)+$ $F(y) F(x) \in Z(R)$ for all $x, y \in R$, then one of the following holds: (1) $R$ contains a nonzero central ideal; (2) $F$ is a left centralizer map of $R$ such that $F: R \rightarrow Z(R)$.

Proof. If we replace $F$ with $-F$ and $d$ with $-d$ in Theorem 3, we conclude that $(-F)(x y)-(-F)(y)(-F)(x) \in Z(R)$ for all $x, y \in R$ implies $x[(-d)(x), x]_{2}=0$ for all $x \in I$, that is, $F(x y)+F(y) F(x) \in Z(R)$ for all $x, y \in R$, implies $x[d(x), x]_{2}=0$ for all $x \in R$. Hence the conclusion follows by Theorem 3 .

We conclude our paper with the following example which shows that the above theorems do not hold for arbitrary rings.

Example: Consider the $\operatorname{ring} R=\left\{\left(\begin{array}{ll}a & b \\ 0 & 0\end{array}\right) \mid a, b \in \mathbb{Z}\right\}$. Obviously, $R$ is not semiprime, because $\left(\begin{array}{ll}0 & 1 \\ 0 & 0\end{array}\right) R\left(\begin{array}{ll}0 & 1 \\ 0 & 0\end{array}\right)=(0)$.

We define maps $F, d: R \rightarrow R$ by $F\left(\begin{array}{cc}a & b \\ 0 & 0\end{array}\right)=\left(\begin{array}{cc}a & 0 \\ 0 & 0\end{array}\right)$ and $d\left(\begin{array}{cc}a & b \\ 0 & 0\end{array}\right)=$ $\left(\begin{array}{cc}0 & -b \\ 0 & 0\end{array}\right)$. Then $F$ is a generalized derivation of $R$ associated with the derivation $d$ of $R$. For $I=R$, we have that $F(x y)-F(x) F(y) \in Z(R)$ for all $x, y \in I$ and $F(x y)-F(y) F(x) \in Z(R)$ for all $x, y \in I$. Since $d(R) \neq(0)$ and $R$ contains no nonzero central ideal for $Z(R)=\left\{\left(\begin{array}{ll}0 & 0 \\ 0 & 0\end{array}\right)\right\}$, the semiprimeness hypothesis in Theorem 1 and Theorem 3 is not superfluous. 


\section{ACKNOWLEDGEMENT}

The authors are thankful to referee for his/her very careful reading of the paper and providing very helpful suggestions and some misprints.

\section{REFERENCES}

[1] E. Albas, "Generalized derivations on ideals of prime rings," Miskolc Mathematical Notes, vol. 14, no. 1, pp. 3-9, 2013.

[2] A. Ali, N. Rehman, and S. Ali, "On lie ideals with derivations as homomorphisms and antihomomorphisms," Acta Math. Hungar, vol. 101, no. 1-2, pp. 79-82, 2003.

[3] S. Ali and S. Huang, "On generalized jordan $(\alpha, \beta)$-derivations that act as homomorphisms or antihomomorphisms," Journal of Algebra and Computational Applications, vol. 1, no. 1, pp. 13-19, 2011, doi: 10.5120/2186-2762.

[4] H. E. Bell and L. C. Kappe, "Rings in which derivations satisfy certain algebraic conditions," Acta Math. Hungar, vol. 53, pp. 339-346, 1989, doi: 10.1007/BF01953371.

[5] H. E. Bell and W. S. Martindale-III, "Centralizing mappings of semiprime rings," Canad. Math. Bull., vol. 30, no. 1, pp. 92-101, 1987, doi: 10.4153/CMB-1987-014-x.

[6] M. N. Daif and H. E. Bell, "Remarks on derivations on semiprime rings," Internat. J. Math. Math. Sci., vol. 15, no. 1, pp. 205-206, 1992, doi: 10.1155/S0161171292000255.

[7] B. Dhara, "Generalized derivations acting as a homomorphism or anti-homomorphism in semiprime rings," Beitr. Algebra Geom., vol. 53, pp. 203-209, 2012, doi: 10.1007/s13366-0110051-9.

[8] B. Dhara and S. Ali, "On $n$-centralizing generalized derivations in semiprime rings with applications to $c^{*}$-algebras," J. Algebra and its Applications, vol. 11, no. 6, pp. Paper No. 1250111,11 pages, 2012, doi: 10.1142/s0219498812501113.

[9] B. Dhara, A. Fosner, and S. Mondal, "Left multipliers on lie ideals in prime rings," Journal of Advanced Research in Pure Mathematics, vol. 5, no. 4, pp. 57-64, 2013.

[10] B. Dhara, S. Sahebi, and V. Rehmani, "Generalized derivations as a generalization of jordan homomorphisms acting on lie ideals and right ideals," Mathematica Slovaca, vol. to appear in No. 4, 2015.

[11] V. D. Filippis, "Generalized derivations as jordan homomorphisms on lie ideals and right ideals," Acta Math. Sinica, English Series, vol. 25, no. 2, pp. 1965-1974, 2009, doi: 10.1007/s10114-0097343-0.

[12] I. Gusic, "A note on generalized derivations of prime rings," Glasnik Mate., vol. 40, no. 60, pp. 47-49, 2005, doi: 10.3336/gm.40.1.05.

[13] I. N. Herstein, Rings with involution. Chicago: Univ. of Chicago Press, 1976.

[14] N. Rehman, "On generalized derivations as homomorphisms and anti-homomorphisms," Glasnik Mate., vol. 39, no. 59, pp. 27-30, 2004, doi: 10.3336/gm.39.1.03.

[15] Y. Wang and H. You, "Derivations as homomorphisms or anti-homomorphisms on lie ideals," Acta Math. Sinica, English Series, vol. 23, no. 6, pp. 1149-1152, 2007, doi: 10.1007/s10114-005-0840$\mathrm{x}$.

Authors' addresses

\section{B. Dhara}

Belda College, Department of Mathematics, Paschim Medinipur, Belda, 721424, INDIA

E-mail address: basu_dhara@yahoo.com 


\section{S. Kar}

Jadavpur University, Department of Mathematics, Kolkata, 700032, INDIA

E-mail address: karsukhendu@yahoo.co.in

K. G. Pradhan

Belda College, Department of Mathematics, Paschim Medinipur, Belda 721424, INDIA

E-mail address: kgp.math@gmail.com 